\title{
Propagating MHD waves in coronal holes
}

\author{
D. Banerjee · G. R. Gupta · L. Teriaca
}

Received: March 15, 2018/ Accepted: date

\begin{abstract}
Coronal holes are the coolest and darkest regions of the solar atmosphere, as observed both on the solar disk and above the solar limb. Coronal holes are associated with rapidly expanding open magnetic fields and the acceleration of the high-speed solar wind. During the years of the solar minima, coronal holes are generally confined to the Sun's polar regions, while at solar maxima they can also be found at lower latitudes. Waves, observed via remote sensing and detected in-situ in the wind streams, are most likely responsible for the wind and several theoretical models describe the role of MHD waves in the acceleration of the fast solar wind. This paper reviews the observational evidences of detection of propagating waves in these regions. The characteristics of the waves, like periodicities, amplitude, speed provide input parameters and also act as constraints on theoretical models of coronal heating and solar wind acceleration.
\end{abstract}

Keywords Sun · Coronal Holes $\cdot$ MHD Waves $\cdot$ Oscillations

\section{Introduction}

Coronal holes are regions of cool and low density plasma that appear 'dark' when seen in lines formed at coronal temperatures (Munro and Withbroe 1972). On the other hand,

D. Banerjee

Indian Institute of Astrophysics, Koramangala, Bangalore 560034, India

Tel.: +918025530672

Fax: +918025534043

E-mail: dipu@iiap.res.in

G. R. Gupta

Indian Institute of Astrophysics, Koramangala, Bangalore, 560034, India

Joint Astronomy Programme, Indian Institute of Science, Bangalore, 560012, India

E-mail: girjesh@iiap.res.in

L. Teriaca

Max-Planck-Institut für Sonnensystemforschung (MPS), 37191, Katlenburg-Lindau, Germany

E-mail: teriaca@linmpi.mpg.de 
they are almost indistinguishable from their surroundings in photospheric and low chromospheric temperatures and there is no significant radiance contrast between the holes and the surrounding region until the temperature exceeds $10^{5} \mathrm{~K}$. During the years of the solar minima, coronal holes are generally confined to the Sun's polar regions, while at solar maxima they can also be found at lower latitudes. Coronal holes are observed both on disk and above the solar limb (off-limb). They are associated with rapidly expanding open magnetic fields and with the acceleration of the high-speed solar wind. For a recent review on coronal holes see Cranmer (2009). During the solar minimum, Ulysses observations clearly show that the solar wind exhibits two modes of outflow: the fast wind, associated with polar coronal holes, with outflow speeds of $\approx 800 \mathrm{~km} \mathrm{~s}^{-1}$ and the slow wind with outflow speeds of $\approx 400 \mathrm{~km} \mathrm{~s}^{-1}$ associated with equatorial regions (Woch et al. 1997; McComas et al. 2000). However, during solar maxima, low latitude coronal holes also show faster than average solar wind speed upto $\approx 600 \mathrm{~km} \mathrm{~s}^{-1}$ (Zhang et al. 2003).

There are several theoretical models which describe the role of MHD waves in the acceleration of the fast solar wind in coronal holes. Some of these models were investigated using 1.5D MHD equations (Boynton and Torkelsson 1996; Lau and Siregar 1996; Stark 1996; Nakariakov et al. 2000; Saito et al. 2001; Lou 2002; Suzuki 2004; Cranmer and van Ballegooijen 2005), 2.5D MHD equations (Ofman and Davila 1995, 1997), 2.5D multifluid MHD equations (Ofman and Davila 2001; Ofman 2004). The common thread in the above models of the fast solar wind acceleration is the required presence of nonlinear MHD waves, or shock wave trains. Some of these wave driven wind models have been reviewed by Ofman (2005).

The detection of these waves in the outer solar atmosphere is made possible by analyzing the effects these waves have on the plasma. The presence or signature of compressional waves may be seen in the form of variations or oscillations in radiance, due to change in plasma density, and also in the line-of-sight (LOS) velocities, due to plasma motions (when they have a significant component directed towards the observer). On the other hand, transverse waves give rise to only LOS effects when they propagate substantially perpendicular to the observer. Moreover the latter give no radiance signature in the theoretical limit of uncompressible Alfvén waves. Temporally and spatially resolved motions result in shifts of the observed profiles while unresolved motions result in broadening of the spectral lines. These effects can be measured from the spectroscopic studies of spectral lines.

Solar and Heliospheric Observatory (SoHO, Domingo et al. 1995) and Hinode (Kosugi et al. 2007) data shed lights onto the dynamical events such as short time scale variability or oscillations in the coronal hole atmosphere observed at Vacuum UltraViolet (VUV: 100 to 1600 ) wavelengths. These periodic oscillations generally carry information from the emitting regions which allows us to diagnose the frozen-in magnetic fields as well as the plasma contained in the different magnetic structures (e.g., plumes, magnetic networks). The wavelengths of these waves are often comparable to the characteristic sizes of these coronal structures and measured time scales are in the range of seconds to few minutes. Table 1 gives the overview of the periodicities and propagation speeds of propagating MHD waves detected in these coronal hole structures. In this review we focus on the observational evidences of waves at different locations in coronal holes (on disk and off-limb) which can put constraints on theoretical models of coronal heating and solar wind acceleration.

\section{Observational evidence of waves in Coronal Holes}

Evidence for Alfvénic fluctuations, and MHD waves is obtained by in situ and remote sensing observations throughout the heliosphere. Here we will describe few representative ob- 
Table 1 Overview of the periodicities and propagation speeds of propagating MHD waves detected in coronal hole structures by remote sensing.

\begin{tabular}{|c|c|c|c|c|}
\hline Authors & Regions & $\begin{array}{l}\text { Periods } \\
\text { (s) }\end{array}$ & $\begin{array}{l}\text { Speed } \\
\left(\mathrm{km} \mathrm{s}^{-1}\right)\end{array}$ & Instrument \\
\hline (Withbroe 1983) & Off-limb & - & $100-200$ & Skylab \\
\hline (Ofman et al. 1997) & Off-limb & 360 & - & UVCS \\
\hline (DeForest and Gurman 1998) & Plumes & $600-900$ & $75-150$ & EIT \\
\hline (Ofman et al. 2000) & Off-limb & $400-625$ & $160-260$ & UVCS \\
\hline (Baneriee et al. 2000) & Plume & $600-1200$ & - & CDS \\
\hline (Banerjee et al. 2001a) & Inter-plume & $1200-1800$ & - & CDS \\
\hline (Banerjee et al. 2001b) & On-disk & $600-1200$ & - & CDS \\
\hline (Marsh et al. 2002) & On-disk & $100-900$ and 1500 & - & CDS \\
\hline (Popescu et al. 2005) & Off-limb & $600-5400$ and 10200 & - & SUMER \\
\hline (O’Shea et al. 2006) & Off-limb & $300-1000$ & $150-170$ & CDS \\
\hline (O’Shea et al. 2007) & On-disk & $300-1000$ & $50-70$ & CDS \\
\hline (Bemporad et al. 2008) & Off-limb & $10000-350000$ & - & UVCS \\
\hline (Tian and Xia 2008) & On-disk & $300-600$ & $30-60$ & TRACE \\
\hline (Gupta et al. 2009) & On-disk & $300-1500$ & $4-15$ & SUMER \\
\hline (Baneriee et al. 2009a) & Plume & $600-1800$ & $75-125$ & EIS-SUMER \\
\hline (Gupta et al. 2010) & Off-limb & $600-1200$ & $25-330$ & EIS-SUMER \\
\hline
\end{tabular}
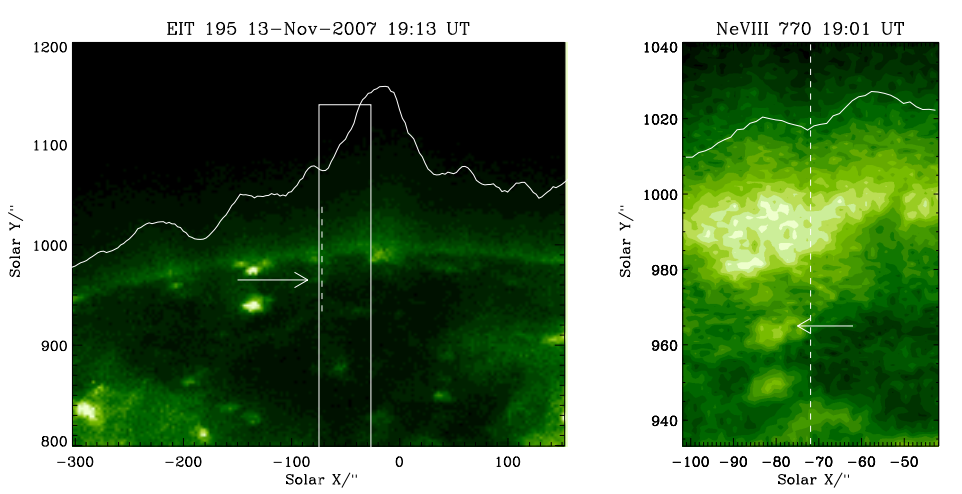

Fig. 1 Left: plume and inter-plume region visible on the EIT image taken on $13^{\text {th }}$ November in the Fe XII 195 passband. The radiance variation along the solar-X is over-plotted as a white line in an arbitrary unit at fixed solar-Y $\approx 1000^{\prime \prime}$. This variation along the solar-X allows to identify the location of plumes and inter-plumes. The rectangular box marks the location of the EIS slot while the dashed line gives the location of the SUMER slit. Right: The SUMER context raster taken in Ne VIII 770 spectral line. The continuous line gives the radiance variation along the solar- $\mathrm{X}$ in an arbitrary unit. In both the panels, the arrow indicates the location of the bright region from where waves are presumably originating. Adapted from Gupta et al. (2010).

servations from low to high heliocentric distances. In the photosphere, the high resolution time series observations of G-band bright points in intergranular lanes show the presence of transverse motions. The mean transverse velocities inferred from these observations can be as large as $1.4 \mathrm{~km} \mathrm{~s}^{-1}$, but only some fraction of that will be likely to propagate upwards on open field lines (e.g., Muller et al. 1994; Nisenson et al. 2003). Banerjee et al. (1998, 2009b) have observed nonthermal broadening of spectral lines from various ions using the SUMER/SoHO (Wilhelm et al. 1995) and EIS/Hinode (Culhane et al. 2007) in off-limb coronal holes. The authors assumed that spectral lines are formed in a plasma with 
an electron temperature $T_{\mathrm{e}}$ equal to the peak temperature of the equilibrium ionization balance for each ion. In this context, broader lines imply an additional unresolved velocity due to wave motions along the line of sight (for further details see section 3.2). At larger heights, measurements of the visible polarization brightness $(\mathrm{pB})$ with the Ultra-Violet Coronagraph Spectrometer (UVCS/SoHO, Kohl et al. 1995) White Light Channel were also used to infer the presence of density oscillations (Ofman et al. 1997). Interplanetary scintillation (IPS) observations of radio signals passing through the corona allow some properties of plasma irregularities to be determined. One way of detecting random fluctuations in the bulk solar wind is by measuring departures from a frozen-in diffraction pattern measured by different sets of radio receivers. Armstrong and Woo (1981) made an early attempt to separate the bulk solar wind flow speed from the random wavelike velocity component within the $30 \mathrm{R} / \mathrm{R}_{\odot}$. Radio IPS measurements are sensitive to density fluctuations over a wide range of spatial scales. Spangler (2002) presented integrated values of $\delta \rho / \rho_{o}$ from VLBI measurements, and compared them to predictions based on specific MHD modes. The Helios 1 and 2 probes uniquely measured the in situ plasma properties between Mercury and the Earth, and they measured MHD fluctuations spanning a wide range of time scales (e.g., Tu and Marsch 1995; Goldstein et al. 1995). The in situ density fluctuation spectra between 0.3 and 1 AU show a large intrinsic variability, with no clear radial trend discernible (Tu and Marsch 1994). Ulysses spacecraft has detected a spectrum of outward propagating and inward propagating waves in the millihertz frequency range with power laws of $f^{-1}$, at low frequency $\left(<10^{-4} \mathrm{~Hz}\right)$ and $f^{-5 / 3}$ for higher frequencies beyond $1 \mathrm{AU}$ (Goldstein et al. 1995)), as well as large amplitude ultra low frequency Alfvén waves (Tsurutani et al. 1995).

\section{Detection of propagating waves in polar plumes and inter-plumes}

The study of polar plumes, the unipolar high density structures in coronal holes, provides clues to the understanding of solar wind acceleration and coronal heating. Plumes subtend an angle of $2^{\circ}$ relative to the Sun centre at low latitude and expand super-radially with the coronal hole (Newkirk and Harvey 1968; DeForest et al. 1997). The region between these structures is termed as inter-plume. From VUV spectroscopy, plumes are known to be denser and cooler than the surrounding inter-plume regions (e.g., Wilhelm 2006), while spectral lines are observed to be broader in inter-plumes (i.e., Banerjee et al. 2000; Giordano et al. 2000; Teriaca et al. 2003). DeForest and Plunkett (1999) produced images from LASCO/SoHO (Brueckner et al. 1995), which clearly show polar plumes extending to altitudes of $25 \mathrm{R} / \mathrm{R}_{\odot}$ or more, very close to the outer edge of the C-3 field of view and above the likely Alfvénic point of the wind flow. Figure 1 shows plume and inter-plume region in coronal hole where the radiance variation along the solar-X at solar- $Y \approx 1000^{\prime \prime}$ is over-plotted as a white line in arbitrary units which allows to identify the location of plumes and inter-plumes in the region within field of view. There is some indication that pressure balance structures which are a common feature observed in situ in high latitude, fast solar wind near solar minimum are remnants of coronal polar plumes (Yamauchi et al. 2002). Plumes are regions of plasma density enhancements. Some of the studies concluded that plumes have lower outflow speeds than inter-plume regions (Noci et al. 1997; Giordano et al. 2000; Wilhelm et al. 2000; Patsourakos and Vial 2000; Teriaca et al. 2003; Raouafi et al. 2007) and, hence, may not contribute significantly to the fast solar wind, whereas some other theoretical and observational studies find higher outflow speeds in plumes than in inter-plume regions for at least some altitudes above the photosphere (Casalbuoni et al. 1999; Gabriel et al. 2003, 2005). These contradictory reports led to the debate on whether plumes or inter-plumes are the pre- 
ferred source regions for the acceleration of the fast solar wind. This topic is highly debated and still open for further confirmation. Polar plumes may also act as Alfvén and slow wave guides. Thus, the study of MHD wave activity in polar plumes is an important and interesting branch of solar coronal physics.
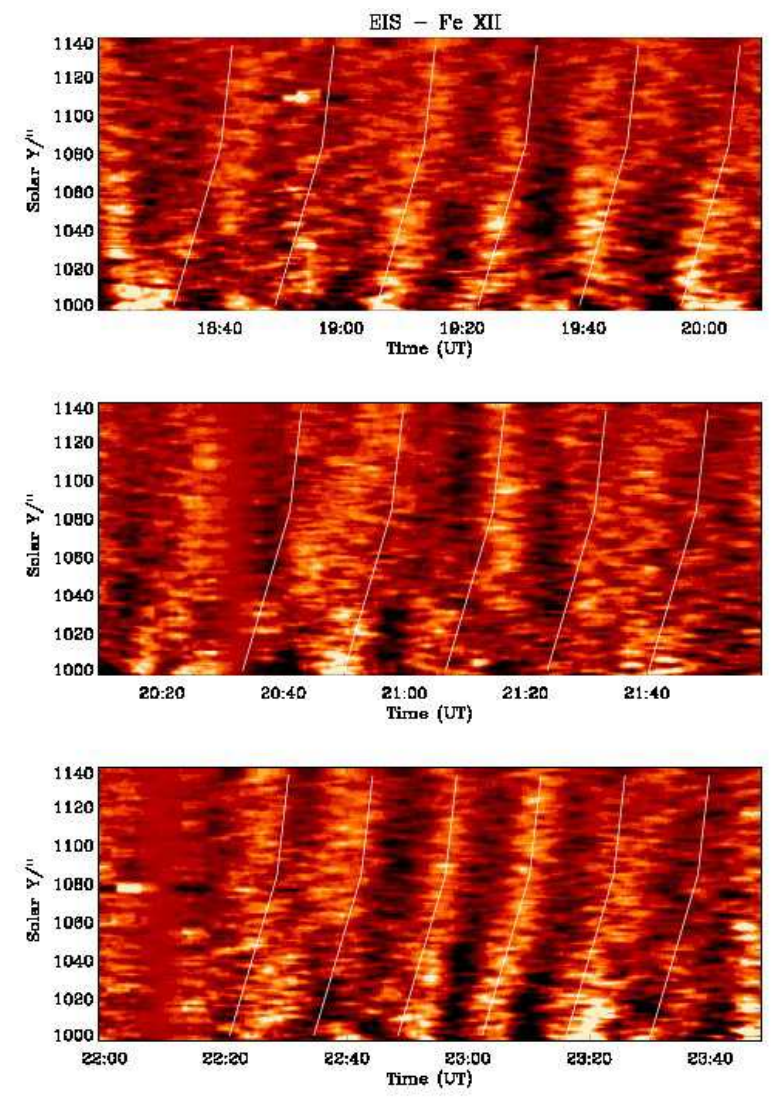

Fig. 2 Enhanced $x$-t map of radiance variation along solar-Y at solar-X $\approx-72^{\prime \prime}$ as recorded by EIS in Fe XII 195 on $13^{\text {th }}$ November 2007. The height range shown here covers the near off-limb and far off-limb region of the North polar coronal hole and corresponds to the inter-plume region. The slanted lines correspond to the disturbances propagating outward with increasing speed. In the near off-limb region the disturbance propagates with speed of $(130 \pm 14) \mathrm{km} \mathrm{s}^{-1}$, and accelerates to $(330 \pm 140) \mathrm{km} \mathrm{s}^{-1}$ in the far off-limb region. The period is in the range of $\approx 15 \mathrm{~min}$ to $18 \mathrm{~min}$. From Gupta et al. (2010).

\subsection{Compressional waves}

The first observational indication of the presence of compressional perturbations in polar plumes was reported by Withbroe (1983) from Skylab observations. Statistically significant short period variations of $\mathrm{Mg}$ X 625 line radiance were detected, with propagating speed in the range of $100 \mathrm{~km} \mathrm{~s}^{-1}$ to $200 \mathrm{~km} \mathrm{~s}^{-1}$ and relative amplitudes of about $10 \%$. Post 

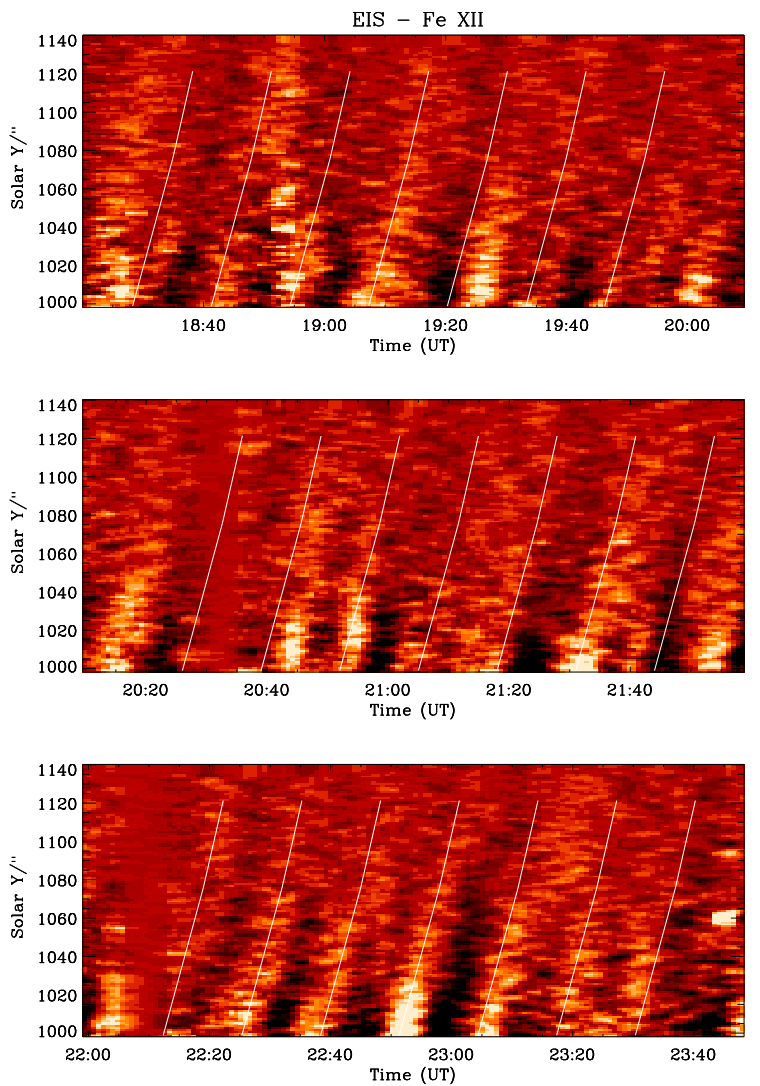

Fig. 3 Enhanced $\mathrm{x}$-t map of radiance variation along solar-Y at solar-X $\approx-39^{\prime \prime}$ as recorded by EIS in Fe XII 195 on $13^{\text {th }}$ November 2007. The height range shown here covers the near off-limb and far off-limb region of the polar coronal hole and falls in the plume region. The slanted lines corresponds to the disturbances propagating outward with nearly constant speed. In the near off-limb region the disturbance propagates with speed of $(135 \pm 18) \mathrm{km} \mathrm{s}^{-1}$, and accelerates to $(165 \pm 43) \mathrm{km} \mathrm{s}^{-1}$ in the far off-limb region. The period is in the range of $\approx 15 \mathrm{~min}$ to $20 \mathrm{~min}$. From Gupta et al. (2010).

SoHO launch, the first undoubted detection of propagating slow MHD waves was made by UVCS/SoHO. Detection of slow waves in an open magnetic structure high above the limb of coronal holes was reported by Ofman et al. (1997, 2000). DeForest and Gurman (1998), analysing Extreme-ultraviolet Imaging Telescope (EIT/SoHO, Delaboudinière et al. 1995) data of polar plumes, detected similar compressive disturbances with relative linear amplitudes of the order of $10 \%$ to $20 \%$ and periods of $10 \mathrm{~min}$ to $15 \mathrm{~min}$. Ofman et al. (1999, 2000) identified the observed compressive longitudinal disturbances as propagating slow MHD waves. Baneriee et al. (2007) have summarized the main features of the observed oscillations within open magnetic structures. A number of studies using Coronal Diagnostic Spectrometer (CDS/SoHO, Harrison et al. 1995) and SUMER/SoHO have reported oscillations in plumes, interplumes and coronal holes in the polar regions of the Sun (e.g., Banerjee et al. 2000, 2001b; O'Shea et al. 2006, 2007) . All of these studies point to the presence of compressional waves, thought to be slow magnetoacoustic waves as found 
by DeForest and Gurman (1998). The detected damping of slow propagating waves was attributed to compressive viscosity.

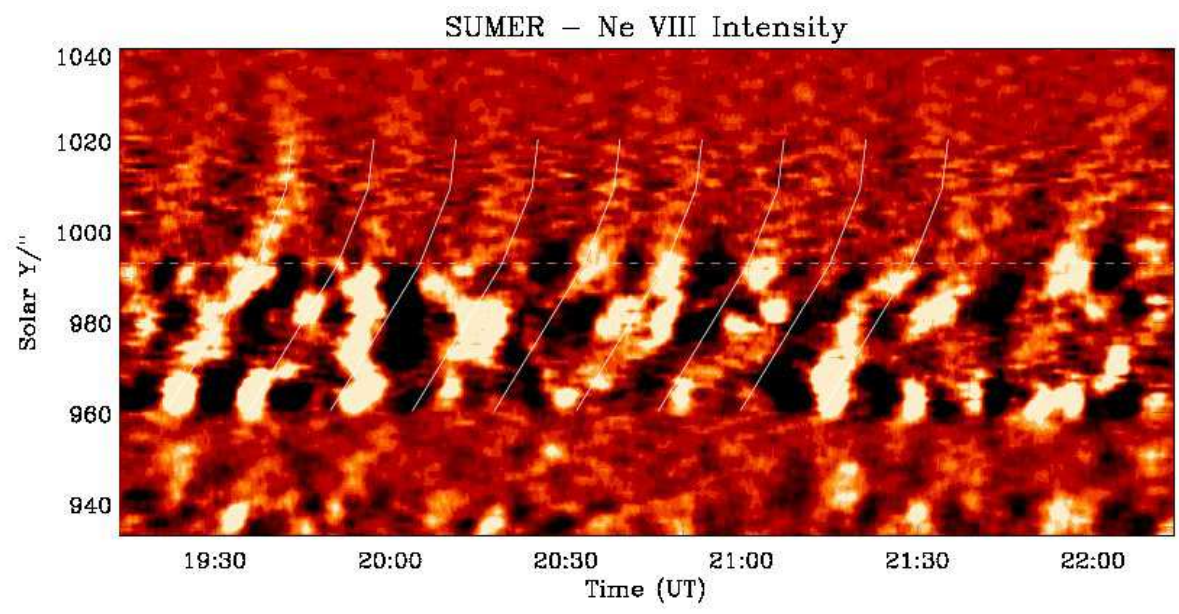

Fig. 4 Enhanced time-distance $\left(\mathrm{x}-\mathrm{t}\right.$ ) map of radiance (along solar-Y) variation at solar $\mathrm{X} \approx-72^{\prime \prime}$ as recorded by SUMER in the Ne VIII 770 spectral line on $13^{\text {th }}$ November 2007. Here the slit covers the on-disk, limb and off-limb region of the polar coronal hole and it is positioned in the inter-plume region. The slanted lines correspond to the disturbances propagating outward with increasing speed. The dashed horizontal line indicates the position of the limb brightening in Ne VIII 770 In the on-disk region the disturbance propagates with a speed of $\approx 25 \pm 1.3 \mathrm{~km} \mathrm{~s}^{-1}$, increasing to $(38 \pm 4.5) \mathrm{km} \mathrm{s}^{-1}$ close to the limb and to about $(130 \pm$ $51) \mathrm{km} \mathrm{s}^{-1}$ in the off-limb region. The period is in the range of $\approx 14 \mathrm{~min}$ to $20 \mathrm{~min}$. From Gupta et al. (2010).

Recently, Gupta et al. (2010) have studied the presence of propagating waves in both the plume and inter-plume region simultaneously. For this study, the data from SUMER and EIS spectrometer has been obtained simultaneously in space and time in several different spectral lines. They have obtained time-distance $(\mathrm{x}-\mathrm{t})$ radiance maps in the Fe XII 195 spectral line in both regions simultaneously. The $\mathrm{x}$ - $\mathrm{t}$ map in inter-plume region shows the signature of acceleration while propagating, see Figure 2 while that for plume region do not show such acceleration, see Figure 3. The measured propagation speed in interplume region was $(130 \pm 14) \mathrm{km} \mathrm{s}^{-1}$ just above the limb, which accelerates to $(330 \pm 140) \mathrm{km} \mathrm{s}^{-1}$ around $160^{\prime \prime}$ above the limb. Whereas in plume region, the propagation speed was in the range of $(135 \pm 18) \mathrm{km} \mathrm{s}^{-1}$ which increases to $(165 \pm 43) \mathrm{km} \mathrm{s}^{-1}$ only. In both the cases, the period of oscillations was in between $15 \mathrm{~min}$ to $20 \mathrm{~min}$. These propagations in inter-plume regions were traced to originate from a bright region of the on-disk part of the coronal hole where the propagation speed is in the range of $(25 \pm 1.3) \mathrm{km} \mathrm{s}^{-1}$ to $(38 \pm 4.5) \mathrm{km} \mathrm{s}^{-1}$ and increases to $(130 \pm 51) \mathrm{km} \mathrm{s}^{-1}$ in off-limb, with the same periodicity, see Figure 4 In this bright region, the oscillations were seen in many spectral lines having a different formation temperature hence different height in the atmosphere. The observed oscillations in different lines have time delays with respect to the oscillations seen in lines forming lower in the atmosphere such as He II 256 and O IV 790 which indicated the presence of upward propagation. From these Gupta et al. (2010) have concluded that these waves were generated somewhere lower in the atmosphere, probably in the chromosphere and then propagate upward and towards the off-limb region. 

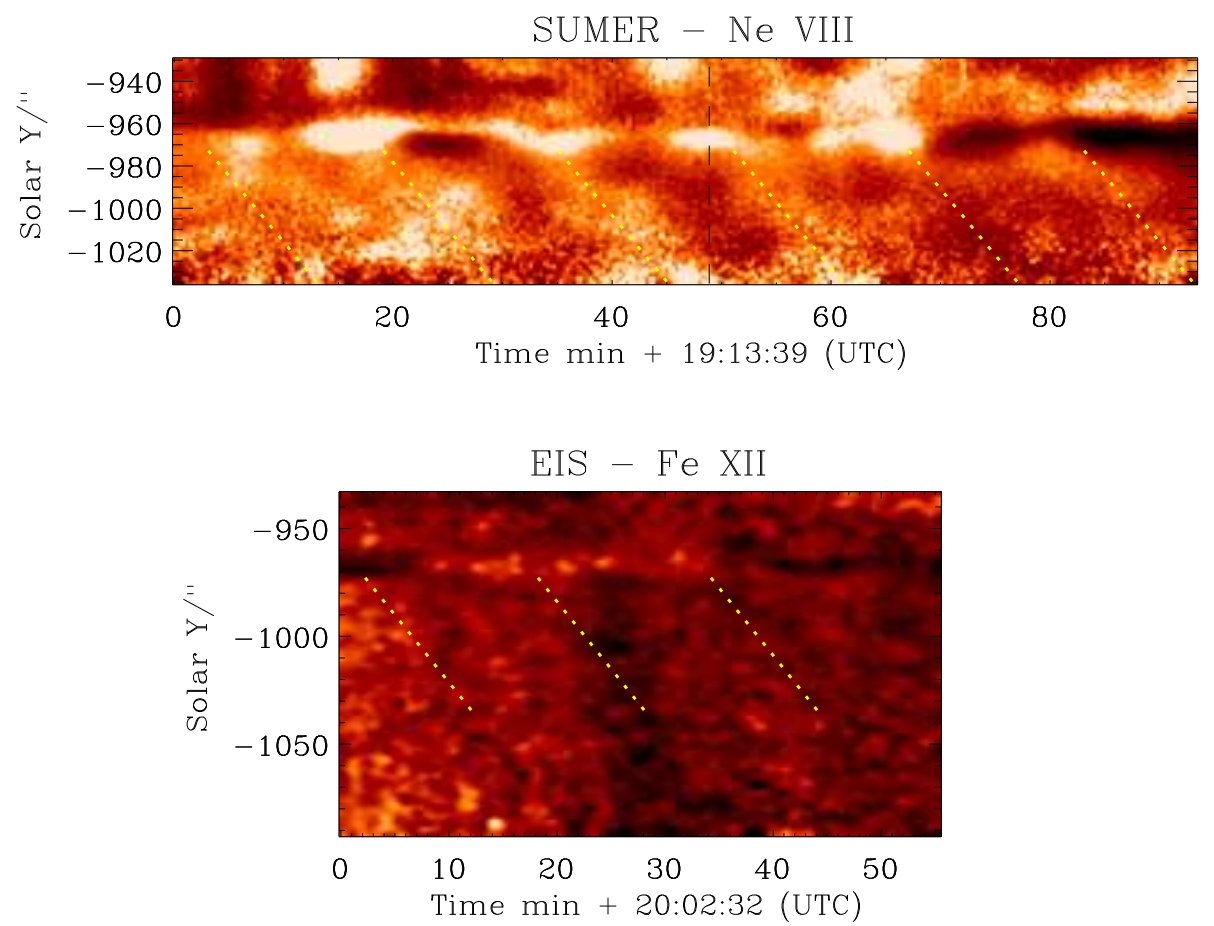

Fig. 5 Enhanced maps of radiance variation along the slit (Solar Y direction) with time for Ne VIII 770 as recorded by SUMER (top panel) and Fe XII 195 as recorded by EIS (bottom panel) on $8^{\text {th }}$ April 2007 for the south polar coronal hole. The vertical black dashed line on the SUMER enhanced radiance map depicts the starting point of the EIS time series (shown in the bottom panel). The slanted dotted yellow lines correspond to disturbances propagating with a speed of $75 \mathrm{~km} \mathrm{~s}^{-1}$ and a period of about $15 \mathrm{~min}$, as determined from the SUMER data. From Baneriee et al. (2009a).

A comparison between the two maps of both off-limb regions, indicate that the waves within the plumes were not observable (may be getting dissipated) far off-limb whereas that was not the case in the inter-plume region. Gupta et al. (2010) have interpreted these waves in inter-plume to be Alfvénic based on the evidence that these obtained propagation speeds were similar in lines formed at different temperature for some overlapping region and the propagation speed profile was found to be roughly consistent with that predicted for Alfvén waves which became supersonic ( $>C_{s} \approx 170 \mathrm{~km} \mathrm{~s}^{-1}$, for Fe XII 195 line formation temperature) in far off-limb region. The presence of oscillations in line width with same range of periodicity as reported were also explained by these Alfvén waves. The radiance oscillations were explained due to the non-linear effects in density stratified atmosphere. Moreover, the measured propagation speeds were also consistent with the fast magnetoacoustic mode of propagation within the error bars of the propagation speeds in Ne VIII 770 and Fe XII 195 lines and can explain the observed radiance oscillations due to its compressible nature. Hence, interpretation of these propagating disturbances in terms of fast magnetoacoustic waves were also indicated/supported. Whereas the propagating waves in plume region were interpreted as slow magneto-acoustic waves. The similar interpretation was also obtained by Banerjee et al. (2009a) for a plume region. Banerjee et al. (2009a) have also studied the 
X-t map obtained from Ne VIII 770 and Fe XII 195 spectral lines simultaneously in plume region, (see Figure 5) and obtained the propagation speed of $75 \mathrm{~km} \mathrm{~s}^{-1}$ and $125 \mathrm{~km} \mathrm{~s}^{-1}$ respectively in both the spectral lines with period of oscillations between $10 \mathrm{~min}$ to $30 \mathrm{~min}$. From these studies, it can be conjectured that the inter-plumes are preferred channel for the acceleration of fast solar wind due to the presence of Alfvén waves which carries energy very far in corona without getting dissipated whereas plumes have slow magneto-acoustic waves which are compressive in nature and can get dissipated by forming shocks in inner corona. Models of waves in plumes show that the waves are damped over a distance of $0.25 \mathrm{R} / \mathrm{R}_{\odot}$ above the photosphere (Cuntz and Suess 2001). In plumes in the lower corona, Coulomb collision are important, and for temperatures of about $1 \mathrm{MK}$ compressive viscosity is an effective dissipation mechanism. Thermal conduction can also contribute significantly to the damping of slow waves in coronal structures.

O'Shea et al. (2006) have studied the propagating waves in off-limb polar coronal holes using CDS/SoHO in a statistical manner (described in section4.1). In that study, they have measured the propagation speed between different line pairs, e.g., O v 629 Mg X 609 \& 625 and Si XII 520 The measured speed between $\mathrm{O}$ V and Mg X lines was (154 \pm 18$) \mathrm{km} \mathrm{s}^{-1}$ whereas that between $\mathrm{O} V$ and Si XII lines was $(218 \pm 28) \mathrm{km} \mathrm{s}^{-1}$. These measured propagation speeds were close to the sound speed for the temperature of formation of these ions, suggesting the presence of slow magneto-acoustic waves. The measured oscillations in their analysis were present over the frequency range of $\approx 0 \mathrm{mHz}$ to $8 \mathrm{mHz}$. They have also reported the evidence of fast magneto-acoustic waves at coronal temperatures based on the histogram of flux-velocity (I-V) phase delay plot. They further conjectured that these waves may have been generated in the upper atmosphere in and around the coronal temperatures at which the $\mathrm{Mg} \mathrm{X}$ and Si XII lines form.

Up to now evidence for the fast magnetoacoustic wave modes in these coronal hole regions has been very limited, even though recent results by Verwichte et al. (2005) have shown that propagating fast magnetoacoustic waves can be present in open magnetic field structures, albeit in this instance, in a post-flare supra-arcade. For the fast mode the wavelengths of the propagating wave should be much shorter than the size of the structure guiding the wave. Shorter wavelength implies shorter period, thus it demands high cadence observations. TRACE can work at $20 \mathrm{~s}$ to $30 \mathrm{~s}$ cadence, allowing us to detect waves with a $40 \mathrm{~s}$ to $60 \mathrm{~s}$ periodicity at the best. Thus, it is difficult to detect smaller periodicity with the present space-based instruments, whereas ground-based coronagraphs and radioheliographs have much better time resolution and have been used for the detection of short-period waves. The Solar Dynamic Observatory should allow us to detect short periodicity with its high cadence imaging instrument AIA.

\subsection{Detection of Alfvén Waves from Variation of Line Width}

As already mentioned, wave-induced motions on unresolved scales result in a broadening of the observed spectral profiles. The measured broadening of the optically thin spectral lines of ions is due to two effects, thermal broadening and non-thermal broadening associated with Doppler shifts due to the unresolved line-of-sight motions

$$
T_{\text {eff }}=T_{i}+\alpha \frac{\mathrm{m}_{\mathrm{i}}}{2 k}<v_{\mathrm{LOS}}^{2}>
$$

where $T_{i}$ is the ion temperature, $k$ is the Boltzmann constant, $v_{\mathrm{LOS}}$ is the line-of-sight component of the velocity, and, $2 / 3 \leq \alpha \leq 1$. There have been several off-limb spectral line 


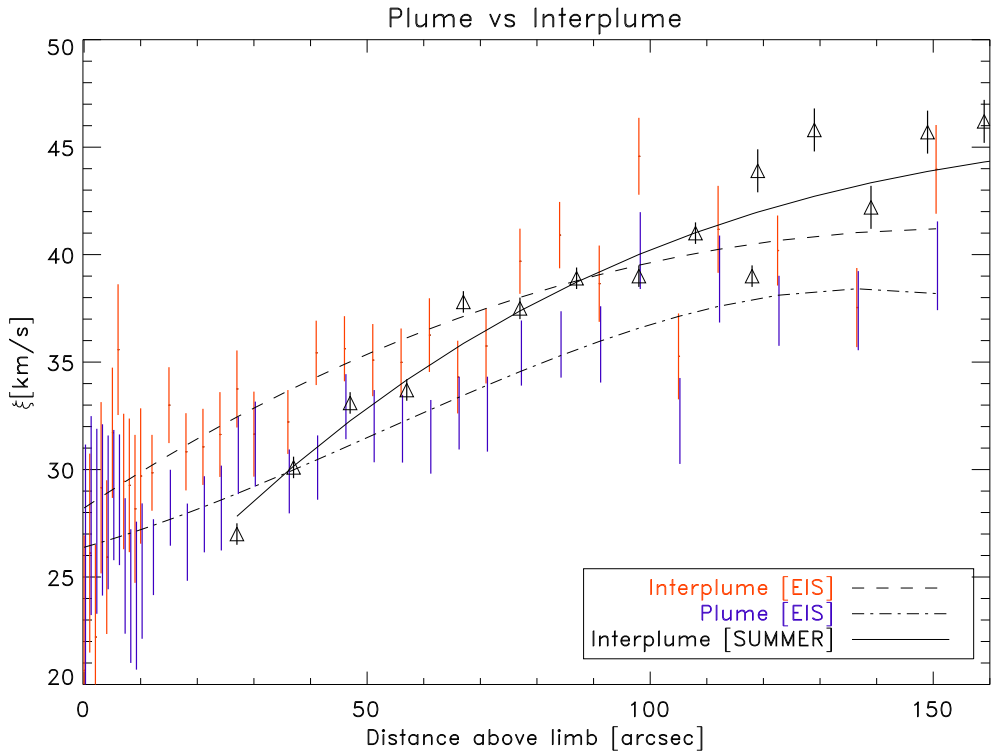

Fig. 6 Variation in nonthermal velocity with height as recorded by Fe XII 195 along a polar plume and interplume. The solid line corresponds to the nonthermal velocity as derived from Si VIII 1446 (Baneriee et al. 1998). The dashed line is a third-order polynomial fit. From Banerjee et al. (2009b)

observations to search for Alfvén wave signatures. Doschek and Feldman (1977) have reported the presence of non-thermal velocities of about $\approx 20 \mathrm{~km} \mathrm{~s}^{-1}$ above the limb $\left(\approx 30^{\prime \prime}\right)$ in coronal hole region in Si VIII 1446 spectral line recorded by the NRL slit spectrograph on Skylab. Measurements of ultraviolet Mg X $609 \& 625$ line widths during a rocket flight showed an increase in width with height to a distance of $70000 \mathrm{~km}$ (Hassler et al. 1990). Also, coronal hole Fe x 6374 spectra taken at Sacramento Peak Observatory showed an increase in the line width with height (Hassler and Moran 1994). SUMER/SoHO were used to record the off-limb, height-resolved spectra of an Si VIII (1446 and 1440 ) density sensitive line pair, in equatorial coronal regions (Dovle et al. 1998; Wilhelm et al. 2005) and in polar coronal holes (Baneriee et al. 1998; Wilhelm et al. 2004). Doyle et al. (1997) and Erdelyi et al. (1998) have also reported the presence of nonthermal velocities in chromospheric, transition region and coronal lines for equatorial region.

The measured variation in line width with density and height supports undamped wave propagation in coronal structures. This was strong evidence of outwardly propagating undamped Alfvén waves in the corona, which may contribute to coronal heating and highspeed solar wind in the case of coronal holes. Alfvén waves propagate virtually undamped through the quasi-static corona and deposit their energy flux in the higher corona. The total energy flux crossing a surface of area $A$ in the corona due to Alfvén waves is given by (Moran 2001)

$$
F=\sqrt{\frac{\rho}{4 \pi}}<\delta v^{2}>B A,
$$

where $\rho$ is the plasma mass density (related to $N_{\mathrm{e}}$ as $\rho \approx m_{\mathrm{p}} N_{\mathrm{e}}, m_{\mathrm{p}}$ is the proton-mass), $<\delta v^{2}>$ is the mean square velocity related to the observed nonthermal velocity as $\xi^{2}=<$ $\delta v^{2}>/ 2$, and $B$, is the magnetic field strength. If the wave energy flux is conserved as the 


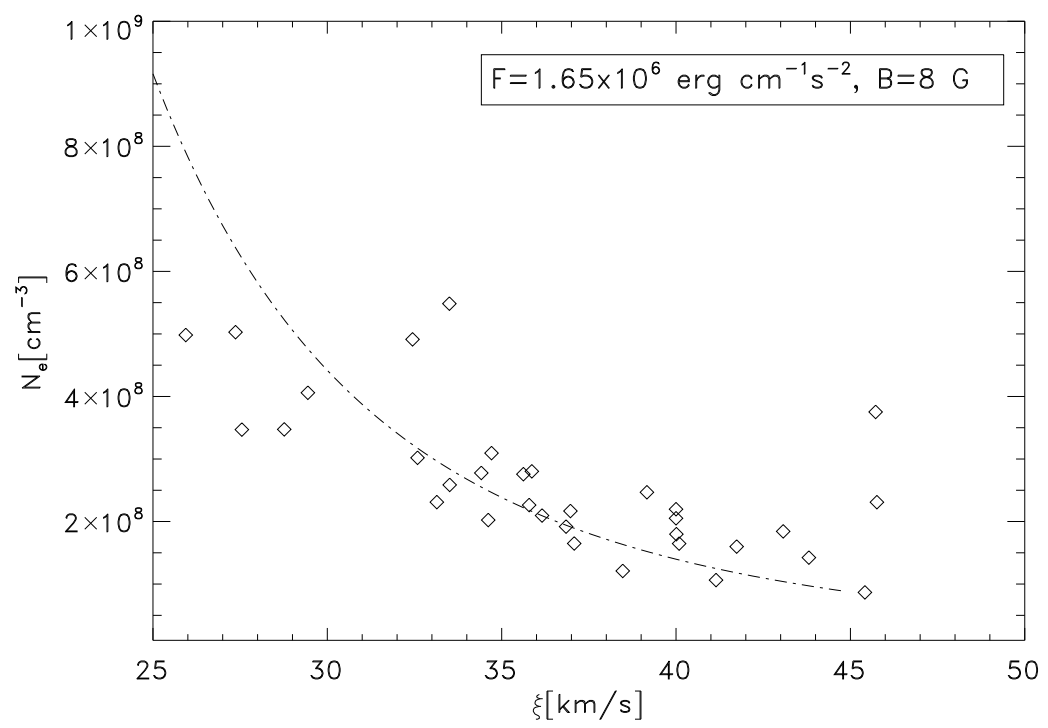

Fig. 7 Variation in electron density with nonthermal velocity for the polar coronal hole. The squared boxes represents the observed values and the solid line represents the theoretical relation (Eq. [4]) for fixed magnetic field strength as indicated. From Banerjee et al. (2009b)

wave propagate outwards, then Eq.(2) gives

$$
<\delta v^{2}>^{1 / 2} \propto \rho^{-1 / 4}(B A)^{-1 / 2}
$$

Now if one assumes a flux tube geometry, then $B A$ is constant with height and Eq.(3) yields

$$
<\delta v^{2}>^{1 / 2} \propto \rho^{-1 / 4} .
$$

To explore the validity of Eq.(4), as done by Banerjee et al. (1998), Banerjee et al. (2009b) plotted Figure 7 corresponding to interplume data. The solid lines are the theoretically predicted functional forms of the variation of number density with nonthermal velocity (Eq.[4]) and the diamonds are the observed data. The proportionality constant have been chosen to match the calculated energy flux. For the interplume data they have used $B=8 \mathrm{G}$ at certain height. Once again the agreement is very good, especially when we are away from the limb. Thus it becomes possible to detect Alfvén waves from the study of the variation of line widths. Dolla and Solomon (2008) have also reported detecting the Alfvén waves from the study of the variation of line widths in solar off-limb region, where they do not find evidence for damping of the Alfvén waves. It will be here interesting to point out that Doyle et al. (1997) have also reported the presence of nonthermal velocities in quiet Sun and coronal hole regions using the fourth rocket flight of High Resolution Telescope and Spectrograph (HRTS-4) instrument. We should also point out that the additional line widths are generaly interpreted in terms of wave-induced motions on unresolved scales but there are two other possibilities; (i) increasing ion temperatures with off-limb distance (Tu et al. 1998), or additional flow components within the FOV due to spicules/macro-spicules flows (Doyle et al. 2005). Are there Alfvén waves present in the lower solar atmosphere? This has been questioned by Erdélyi and Fedun (2007) while looking at the initial results from 


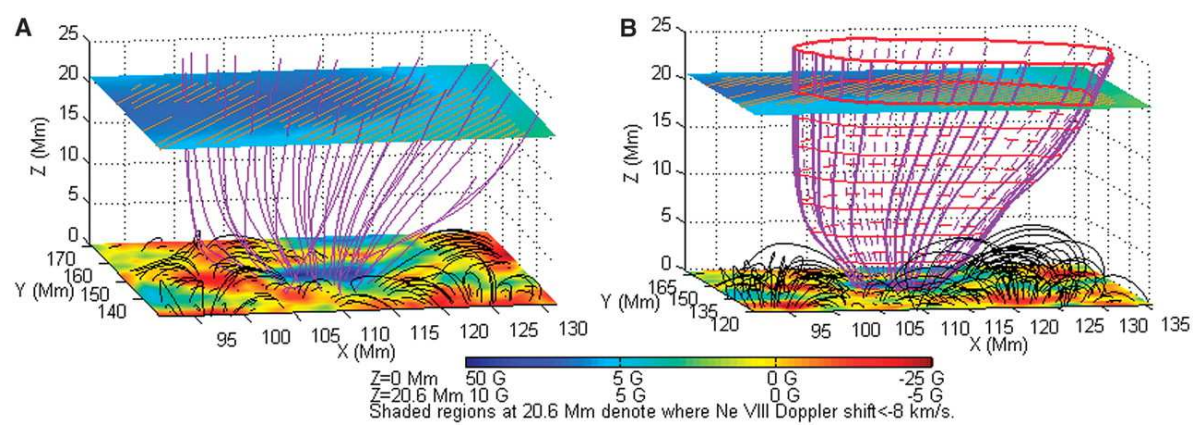

Fig. 8 Magnetic funnel in the solar atmosphere. (A) Emphasis on open field lines and correlation with the Ne VIII 770 outflow speed larger than $8 \mathrm{~km} \mathrm{~s}^{-1}$ (dark shading). (B) Illustration of the funnel boundary and magnetic unipolar flux constriction by adjacent, surrounding bipolar loops. From Tu et al. (2005)

Hinode. Jess et al. (2009) reported the detection of oscillatory phenomena of line widths (FWHM) associated with a large bright point group located near the solar disk center which indicates the presence of Alfvén waves in that region. These studies have focussed on the detection of the oscillatory nature of the line widths at fixed location rather than variation with height.

\section{Propagating Waves in on-disk region of coronal holes}

In the previous sections we have discussed the observational evidences of the presence of propagating MHD waves. Now we would look for their origin. These waves must be generated somewhere within the disk part of the coronal hole. Similar to the quiet Sun, a network and internetwork pattern can also be seen all over the coronal holes in chromospheric and transition region lines. The magnetic field is predominantly concentrated on these network regions and the footpoints of coronal funnels emanate from these regions, see Figure 8 (Tu et al. 2005). All the open field lines forming the coronal holes essentially originate from such regions and the fast solar winds emanate from these vertical flux tubes. Tsuneta et al. (2008) have used the high spatial resolution of Solar Optical Telescope aboard Hinode to characterize the magnetic landscape of the Sun's polar region. They have conjectured that the vertical flux tubes originating from the kilogauss magnetic patches in coronal holes will fan out in the lower atmosphere of the coronal holes. All these patches have the same polarity which is consistent with the global polarity of the polar region. They have further conjectured that these vertical flux tubes with large expansion around the photospheric-coronal boundary (which may be the upper chromosphere) will serve as efficient chimneys for the propagation of Alfvén waves which accelerate the solar wind. The location of these magnetic patches is still unclear but most likely, are placed on network boundaries within the coronal holes. The nature of waves in these regions are being studied now and some recent results are discussed in the next section.

\subsection{Detection of Waves using statistical techniques}

Gupta et al. (2009) had studied the nature of propagating waves in these network and internetwork regions using SUMER polar coronal hole data in a statistical manner. They have 
divided the dataset in those two regions based on the intensity enhancements. They have seen the similar kind of oscillations in two spectral lines of N IV 765 and Ne VIII 770 in intensity as well as in velocity. Phase delays were measured using the technique of Athay and White (1979) and plotted the phase delays over the full range of $-180^{\circ}$ to $180^{\circ}$ as a function of frequency. An example of the result of this is shown in Figure 9 from Gupta et al. (2009).

In this Figure, the phase delays measured between the line pair for both the network and internetwork regions are shown. From Figure 9, it can be seen that the measured oscillations are present over the frequency range of $\approx 0 \mathrm{mHz}$ to $8 \mathrm{mHz}$. It can be also seen that these phases line up along parallel straight lines. This distribution of phases along straight lines indicates the presence of upward or downward propagating waves depending upon the inclination of straight line. From the measurement of slope of these lines one can obtain the time delays between the different lines, using the phase equation,

$$
\Delta \phi=2 \pi f T
$$

where $f$ is the frequency and $\mathrm{T}$ the time delay in seconds, the phase difference will vary linearly with $f$, and will change by 360 over frequency intervals of $\Delta f=1 / T$. This will give rise to parallel lines in $\Delta \phi$ vs. $f$ plots at fixed frequency intervals $(\Delta f=1 / T)$, corresponding to a fixed time delay T. In the case of Figure 9 The phase plot for network region (left panel) shows only the presence of upwardly propagating waves whereas that for internetwork region (right panel) shows the presence of both upward and downward propagating waves. The time delay measured for the two spectral lines was found to be $(717 \pm 114) \mathrm{s}$ for the network regions whereas that for the internetwork regions were $(216 \pm 38) \mathrm{s}$ and $(778 \pm 133) \mathrm{s}$ for the upwardly and downwardly propagating waves respectively. Using the limb-brightening technique, the height difference between the spectral lines were measured and using these time delays, Gupta et al. (2009) have calculated the upwardly propagation speed of $(4.0 \pm 0.6) \mathrm{km} \mathrm{s}^{-1}$ in network region whereas upwardly and downwardly propagation speeds were $(13.2 \pm 2.3) \mathrm{km} \mathrm{s}^{-1}$ and $(3.7 \pm 0.5) \mathrm{km} \mathrm{s}^{-1}$ respectively in internetwork region. These measured speeds suggest the presence of slow magneto-acoustic waves in these on-disk regions of coronal hole. Gupta et al. (2009) have conjectured that the internetwork regions within coronal holes are composed of low-lying coronal loops, where waves can travel both upwardly and downwardly, whereas the network regions are filled with more open, funnel like structures where only upward propagation is possible (see Figure 8). It has also been point out that statistically there were more velocity oscillations than intensity oscillations in the phase plots. For compressional types of waves, signatures are seen in both. So if there are only velocity oscillations present in some locations this implies the presence of transverse-type waves, like Alfvénic. It has been further pointed out that in this particular study the identified network region spans a large spatial domain along the slit, which would be due to the bigger magnetic patch. As found in Gupta et al. (2010) (see also section 3), the waves propagating in the off-limb region which has its origin in on-disk bright region can also be linked to the magnetic patch on network boundary. The nature of oscillation observed on that region is very much similar to that observed by Gupta et al. (2009) in this case. Figure 10 shows the kind of oscillations observed by Gupta et al. (2010) in the bright region in the Ne VIII 770 spectral line radiance. The similar oscillations are also present in Ne VIII 770 Doppler velocity. The generation mechanism of these oscillations is still unclear. Overall these regions are very much similar to the magnetic patches reported by Tsuneta et al. (2008) and hence will serve as the efficient chimneys for Alfvén waves which will accelerate the solar wind. 

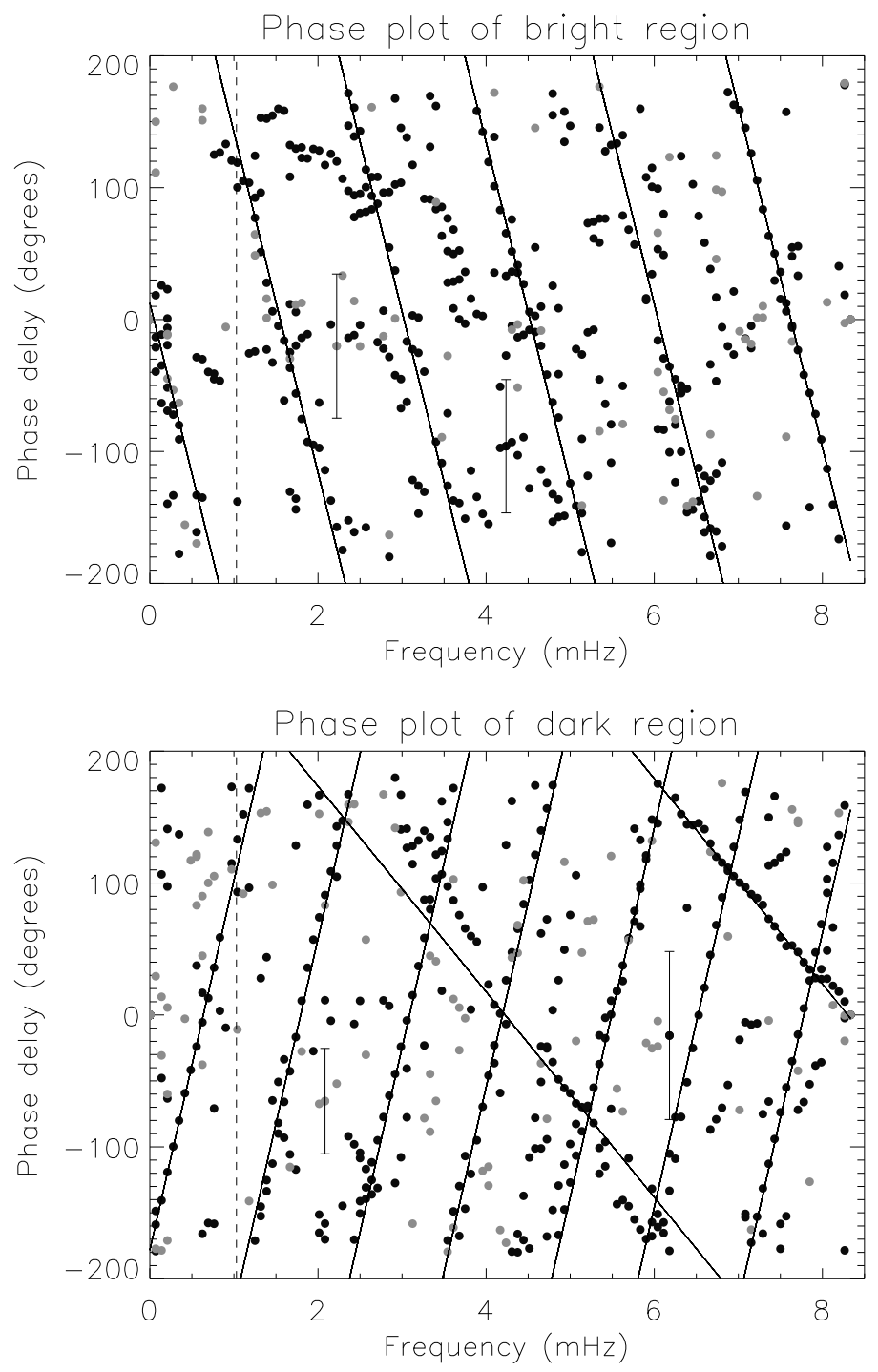

Fig. 9 Phase delays measured between the oscillations in the spectroscopic line pair for the bright (top panel) and dark (bottom panel) locations. Presumably, the bright locations correspond to the network boundaries. The phases in radiant flux oscillations are shown in the grey circle symbols while that in LOS velocities are shown as the black circle symbols. Overplotted on each Figure are black parallel lines, corresponding to fixed time delays. The vertical dashed line drawn at $1.03 \mathrm{mHz}$ indicates that some phase values below this could be affected by solar rotation. Representative errors on the phase measurements are indicated by the error bars. From Gupta et al. (2009). 


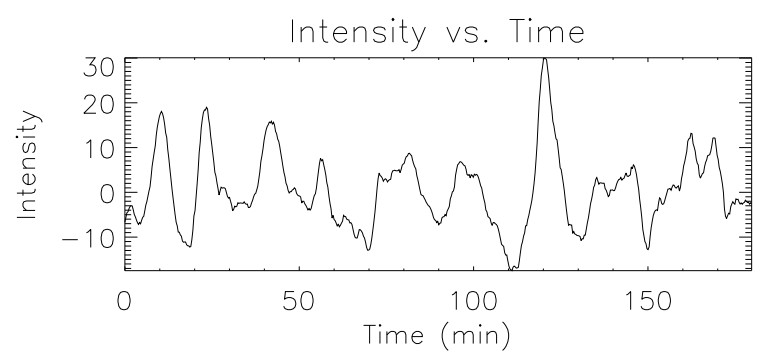

Global Period at max. power $(<65.5 \mathrm{~min}$.

$\mathrm{P} 1=19.84 \mathrm{~min}$.
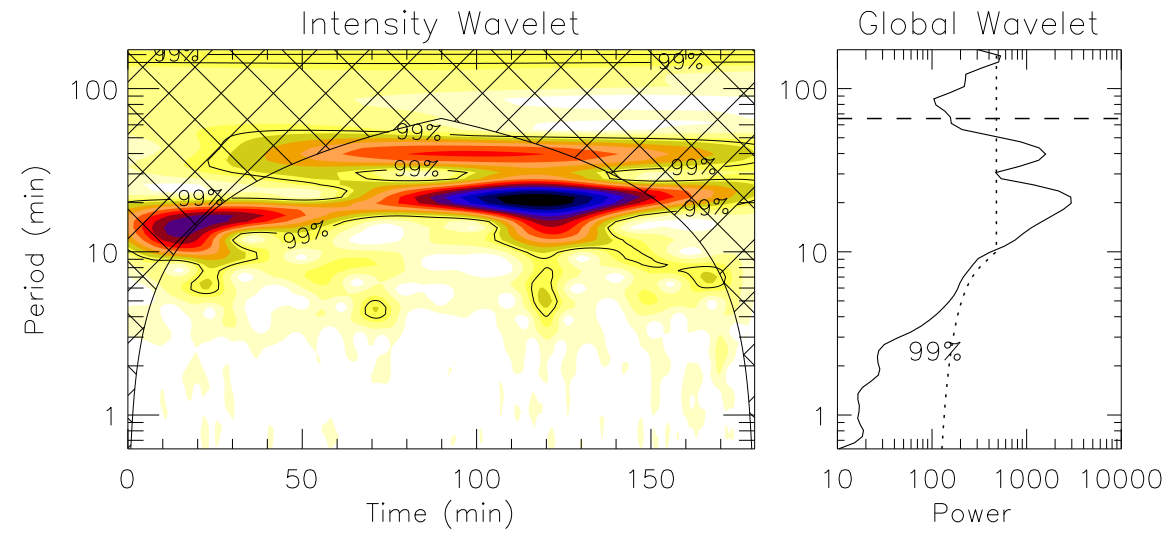

Fig. 10 The wavelet result for the on-disk location at solar-Y $\approx 967^{\prime \prime}$ and solar-X $\approx-72^{\prime \prime}$ in Ne VIII radiance (left side) and velocity (right side). The top panel shows the relative (background trend removed) radiance smoothed over 3 min. Bottom left panel shows the color inverted wavelet power spectrum with $99 \%$ confidence level contours while bottom right panel show the global (averaged over time) wavelet power spectrum with $99 \%$ global confidence level drawn. The period P1 at the location of the maximum in the global wavelet spectrum is printed above the global wavelet spectrum. From Gupta et al. (2010).

\subsection{Waves in equatorial coronal holes}

So far we were discussing on polar coronal holes. Coronal holes are often found, particularly during solar maximum in the equatorial region also. Tian and Xia (2008) have studied the network oscillations in equatorial coronal hole. For the analysis, two bandpass (1600 and 171 ) time series images were obtained from the TRACE spacecraft. They have measured the oscillations of period in between $5 \mathrm{~min}$ to $10 \mathrm{~min}$ in network enhanced regions from wavelet analysis. Using the time-distance map for the same region, the propagation speeds were measured to be $32 \mathrm{~km} \mathrm{~s}^{-1}$ for the 10 min oscillation and $58 \mathrm{~km} \mathrm{~s}^{-1}$ for the $5 \mathrm{~min}$ oscillation, see Figure 11 Tian and Xia (2008) have interpreted these propagations to be slow magneto-acoustic waves and calculated the energy flux of $40 \mathrm{erg} \mathrm{cm}^{-2} \mathrm{~s}^{-1}$ for the quiet coronal conditions which are much lower than the required heat input to the quiet corona. Whereas for the chromospheric conditions, the calculated energy flux is $1.368 \times 10^{6}$ erg cm$~_{-2} \mathrm{~s}^{-1}$ which is almost one third of the required energy budget for the chromosphere. O'Shea et al. (2007) have also studied the propagating waves in equatorial as well as in polar coronal holes using CDS/SoHO. They have also analyzed these propagating waves in a statistical manner which is explained in section 4.1 In their study, they have measured the propagation speed between different line pairs, e.g., O v 629 Mg X 624 and Si XII 520 . The obtained propagation speed between these line pairs was in between $50 \mathrm{~km} \mathrm{~s}^{-1}$ to 

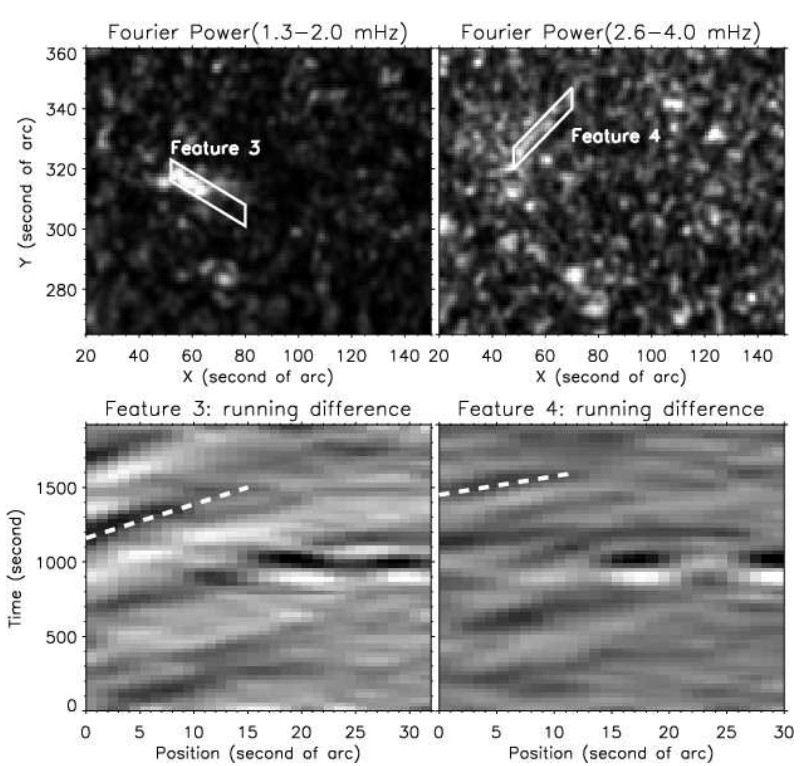

Fig. 11 Upper: Maps of Fourier power in frequency ranges of $1.3 \mathrm{mHz}$ to $2.0 \mathrm{mHz}$ and $2.6 \mathrm{mHz}$ to $4.0 \mathrm{mHz}$ for the 171 passband. Lower: plots of running differences along the long sides of the bars shown in the upper panels. The dashed lines used to calculate the speed of propagating oscillations. From Tian and Xia (2008).

$120 \mathrm{~km} \mathrm{~s}^{-1}$ and the measured oscillations were present over the frequency range of $\approx 0 \mathrm{mHz}$ to $8 \mathrm{mHz}$. They have estimated the energy carried by these waves in WKB approximation, and obtained the wave energy flux of $\approx 2.5 \times 10^{4} \mathrm{erg} \mathrm{cm}^{-2} \mathrm{~s}^{-1}$. This is lower than the energy flux requirement of a coronal hole with the high speed wind $\left(\approx 8 \times 10^{5} \mathrm{erg} \mathrm{cm}^{-2} \mathrm{~s}^{-1}\right)$, but non-WKB effects may enhance the energy flux somewhat. O'Shea et al. (2007) have also found that these waves were propagating preferentially in regions of increased brightness in the coronal holes that are considered to be locations of concentrated magnetic field which could be the high field magnetic patches as reported by Tsuneta et al. (2008).

\subsection{The source of the propagating waves}

In order to answer the question of where propagating coronal waves originate from, De Pontieu et al. (2004); De Pontieu and Erdélyi (2006) developed the general framework of how photospheric oscillations can leak into the atmosphere along inclined magnetic-flux tubes. In a non-magnetic atmosphere $p$ modes are evanescent and cannot propagate upwards through the temperature minimum barrier since their period $P(\approx 200 \mathrm{~s}$ to $450 \mathrm{~s})$ is above the local acoustic cut-off period $P_{c} \approx 200 \mathrm{~s}$. However, in a magnetically structured atmosphere, where the field lines have some natural inclination $(\theta)$, where $\theta$ is measured between the magnetic guide channelling the oscillations and the vertical, the acoustic cut-off period takes the form $P_{c} \sim \sqrt{T} / \cos \theta$ with the temperature $T$. This inclination will allow some nonpropagating evanescent wave energy to tunnel through the temperature minimum into the hot chromosphere of the waveguide, where propagation is once again possible because of higher temperatures $\left(P_{c}>300 \mathrm{~s}\right)$. There has been recent attempts to numerically model the direct propagation of acoustic waves, driven harmonically at the solar photosphere, into the three-dimensional solar atmosphere in the framework of ideal magnetohydrodynamics 
(Erdélyi et al. 2007; Malins and Erdélyi 2007; Fedun et al. 2009). They have studied the leakage of $5 \mathrm{~min}$ global solar acoustic oscillations into the upper, gravitationally stratified and magnetised atmosphere, where the modelled solar atmosphere possesses realistic temperature and density stratification. They have shown that high-frequency waves can propagate from the lower atmosphere across the transition region, experiencing relatively low reflection, and transmitting most of their energy into the corona. The thin transition region acts as a wave guide for horizontally propagating surface waves for a wide range of driver periods. One must point out that this modelling has been restricted to either for simple extended flux tubes or non magnetic atmosphere. More realistic models are needed to verify some of these calculations.

\section{Concluding remarks}

There is direct evidence of the presence of magnetoacoustic waves in solar coronal holes obtained with EUV imagers and spectroscopy (time-distance radiance maps). The wave relative amplitudes are several percent and periods are several minutes. Evidence for Alfvén and fast magnetoacoustic waves is indirect but abundant in both imaging and spectral data. The source of these waves and the physical mechanism responsible for the observed periodicity has not yet been fully understood. Theoretical progress in this field is very much needed to explain the observed signatures.

Recently McIntosh et al. (2010) have analyzed STEREO observations of plumes and interpreted them in terms of quasi-periodically forced jets. In almost all polar plumes observed at the limb in these STEREO sequences, in all coronal passbands, they observe radiance variations travelling along the structures with a mean velocity of $135 \mathrm{~km} \mathrm{~s}^{-1}$ at a range of temperatures from 0.5 MK to 1.5 MK, that they interpret as high speed jets of plasma. The jets have an apparent brightness enhancement of $5 \%$ above that of the plumes they travel on and repeat quasi-periodically, with repeat-times ranging from five to twenty-five minutes. They also claim that these perturbations have very similar properties to those observed in the quiet Sun by McIntosh and De Pontieu (2009). In each case, these perturbations have been connected spectroscopically to a strong upflowing, weak emission component at the magnetic footpoints. The spectroscopically determined upflows appear to be rooted in dynamic Type-II spicules in the upper chromosphere (De Pontieu et al. 2009). It should be pointed out here that this alternative interpretation is contrary to the widely held interpretation that this observational phenomenon is due to compressive waves, nonetheless it should not be ruled out completely but a further analysis and new set of observation in equatorial coronal hole is desired (as indicated by McIntosh et al. (2010)) to address the wave/upflow interpretation issue. Often the non-detection of radiance variations at larger heights are due to poor signal to noise, so future imaging instruments with better detectors can also address this issue. One should also consider Doppler dimming results from UVCS/SoHO and SUMER/SoHO, which shows that velocities above $100 \mathrm{~km} \mathrm{~s}^{-1}$ are reached only above $1.5 \mathrm{R} / \mathrm{R}_{\odot}$ in either plumes or interplumes (Teriaca et al. 2003; Gabriel et al. 2003, 2005). Besides, there is no evidence of such large flows in accurate Doppler maps of the off-limb region of coronal holes (Wilhelm et al. 1998; Teriaca 2010).

The future of wave detection looks very promising . With the Hinode satellite, containing the X-Ray Telescope (XRT) and the EUV Imaging Spectrometer (EIS), and the just launched Solar Dynamics Observatory (SDO), containing the Atmospheric Imaging Assembly (AIA), means that it will soon be possible to obtain slit and image data at a much increased spectral resolution with excellent time resolution. For example, the AIA, offering a replacement for 
the TRACE instrument, will allow the Sun to be imaged at ten different wavelengths, eight of them simultaneously, with a time resolution of $\approx 10 \mathrm{~s}$. The good spectral resolution of EIS will, in addition, allow the accurate measurement of non-thermal velocities and allow other studies that are based on the detailed measurement of line widths, together with a simultaneous measurement of electron density in these structures.

Acknowledgements We like to thank Prof. Klaus Wilhelm for careful reading of the manuscript.

\section{References}

J.W. Armstrong, R. Woo, Solar wind motion within 30 R solar masses - Spacecraft radio scintillation observations. Astron. Astrophys. 103, 415-421 (1981)

R.G. Athay, O.R. White, Chromospheric oscillations observed with OSO 8. IV - Power and phase spectra for C IV. Astrophys. J. 229, 1147-1162 (1979). doi:10.1086/157050

D. Banerjee, E. O'Shea, J.G. Doyle, Long-Period Oscillations in Polar Plumes as Observed by cds on Soho. Solar Phys. 196, 63-78 (2000)

D. Banerjee, D. Pérez-Suárez, J.G. Doyle, Signatures of Alfvén waves in the polar coronal holes as seen by EIS/Hinode. Astron. Astrophys. 501, 15-18 (2009b). doi:10.1051/0004-6361/200912242

D. Banerjee, L. Teriaca, J.G. Doyle, K. Wilhelm, Broadening of SI VIII lines observed in the solar polar coronal holes. Astron. Astrophys. 339, 208-214 (1998)

D. Banerjee, L. Teriaca, J.G. Doyle, P. Lemaire, Polar Plumes and Inter-plume regions as observed by SUMER on SOHO. Solar Phys. 194, 43-58 (2000)

D. Banerjee, E. O'Shea, J.G. Doyle, M. Goossens, Long period oscillations in the inter-plume regions of the Sun. Astron. Astrophys. 377, 691-700 (2001a). doi:10.1051/0004-6361:20011153

D. Banerjee, E. O'Shea, J.G. Doyle, M. Goossens, Signatures of very long period waves in the polar coronal holes. Astron. Astrophys. 380, 39-42 (2001b). doi:10.1051/0004-6361:20011548

D. Banerjee, R. Erdélyi, R. Oliver, E. O’Shea, Present and Future Observing Trends in Atmospheric Magnetoseismology. Solar Phys. 246, 3-29 (2007). doi:10.1007/s11207-007-9029-z

D. Banerjee, L. Teriaca, G.R. Gupta, S. Imada, G. Stenborg, S.K. Solanki, Propagating waves in polar coronal holes as seen by SUMER and EIS. Astron. Astrophys. 499, 29-32 (2009a). doi:10.1051/0004$6361 / 200912059$

A. Bemporad, W.H. Matthaeus, G. Poletto, Low-Frequency Ly $\alpha$ Power Spectra Observed by UVCS in a Polar Coronal Hole. Astrophys. J. Lett. 677, 137-140 (2008). doi:10.1086/588093

G.C. Boynton, U. Torkelsson, Dissipation of non-linear Alfven waves. Astron. Astrophys. 308, 299-308 (1996)

G.E. Brueckner, R.A. Howard, M.J. Koomen, C.M. Korendyke, D.J. Michels, J.D. Moses, D.G. Socker, K.P. Dere, P.L. Lamy, A. Llebaria, M.V. Bout, R. Schwenn, G.M. Simnett, D.K. Bedford, C.J. Eyles, The Large Angle Spectroscopic Coronagraph (LASCO). Solar Phys. 162, 357-402 (1995). doi:10.1007/BF00733434

S. Casalbuoni, L. Del Zanna, S.R. Habbal, M. Velli, Coronal plumes and the expansion of pressure-balanced structures in the fast solar wind. J. Geophys. Res. 104, 9947-9962 (1999). doi:10.1029/1999JA900047

S.R. Cranmer, Coronal Holes. Living Reviews in Solar Physics 6, 3 (2009)

S.R. Cranmer, A.A. van Ballegooijen, On the Generation, Propagation, and Reflection of Alfvén Waves from the Solar Photosphere to the Distant Heliosphere. Astrophys. J. Suppl. 156, 265-293 (2005). doi:10.1086/426507

J.L. Culhane, L.K. Harra, A.M. James, K. Al-Janabi, L.J. Bradley, R.A. Chaudry, K. Rees, J.A. Tandy, P. Thomas, M.C.R. Whillock, B. Winter, G.A. Doschek, C.M. Korendyke, C.M. Brown, S. Myers, J. Mariska, J. Seely, J. Lang, B.J. Kent, B.M. Shaughnessy, P.R. Young, G.M. Simnett, C.M. Castelli, S. Mahmoud, H. Mapson-Menard, B.J. Probyn, R.J. Thomas, J. Davila, K. Dere, D. Windt, J. Shea, R. Hagood, R. Moye, H. Hara, T. Watanabe, K. Matsuzaki, T. Kosugi, V. Hansteen, Ø. Wikstol, The EUV Imaging Spectrometer for Hinode. Solar Phys. 243, 19-61 (2007). doi:10.1007/s01007-007-0293-1

M. Cuntz, S.T. Suess, Shock Formation of Slow Magnetosonic Waves in Coronal Plumes. Astrophys. J. Lett. 549, 143-146 (2001). doi:10.1086/319147

B. De Pontieu, R. Erdélyi, The nature of moss and lower atmospheric seismology. Royal Society of London Philosophical Transactions Series A 364, 383-394 (2006)

B. De Pontieu, R. Erdélyi, S.P. James, Solar chromospheric spicules from the leakage of photospheric oscillations and flows. Nature 430, 536-539 (2004). doi:10.1038/nature02749 
B. De Pontieu, S.W. McIntosh, V.H. Hansteen, C.J. Schrijver, Observing the Roots of Solar Coronal Heating in the Chromosphere. Astrophys. J. Lett. 701, 1-6 (2009). doi:10.1088/0004-637X/701/1/L1

C.E. DeForest, J.B. Gurman, Observation of Quasi-periodic Compressive Waves in Solar Polar Plumes. Astrophys. J. Lett. 501, 217 (1998). doi:10.1086/311460

C.E. DeForest, S.P. Plunkett, Polar Plumes Observed at Extreme Coronal Altitudes, in American Astronomical Society Meeting Abstracts. American Astronomical Society Meeting Abstracts, vol. 194, 1999, pp. 10801

C.E. DeForest, J.T. Hoeksema, J.B. Gurman, B.J. Thompson, S.P. Plunkett, R. Howard, R.C. Harrison, D.M. Hassler, Polar Plume Anatomy: Results of a Coordinated Observation. Solar Phys. 175, 393-410 (1997). doi:10.1023/A:1004955223306

J. Delaboudinière, G.E. Artzner, J. Brunaud, A.H. Gabriel, J.F. Hochedez, F. Millier, X.Y. Song, B. Au, K.P. Dere, R.A. Howard, R. Kreplin, D.J. Michels, J.D. Moses, J.M. Defise, C. Jamar, P. Rochus, J.P. Chauvineau, J.P. Marioge, R.C. Catura, J.R. Lemen, L. Shing, R.A. Stern, J.B. Gurman, W.M. Neupert, A. Maucherat, F. Clette, P. Cugnon, E.L. van Dessel, EIT: Extreme-Ultraviolet Imaging Telescope for the SOHO Mission. Solar Phys. 162, 291-312 (1995). doi:10.1007/BF00733432

L. Dolla, J. Solomon, Solar off-limb line widths: Alfvén waves, ion-cyclotron waves, and preferential heating. Astron. Astrophys. 483, 271-283 (2008). doi:10.1051/0004-6361:20077903

V. Domingo, B. Fleck, A.I. Poland, The SOHO Mission: an Overview. Solar Phys. 162, 1-37 (1995). doi:10.1007/BF00733425

G.A. Doschek, U. Feldman, The coronal temperature and nonthermal motions in a coronal hole compared with other solar regions. Astrophys. J. Lett. 212, 143-146 (1977). doi:10.1086/182394

J.G. Doyle, D. Banerjee, M.E. Perez, Coronal line-width variations. Solar Phys. 181, 91-101 (1998)

J.G. Doyle, E. O'Shea, R. Erdelyi, K.P. Dere, D.G. Socker, F.P. Keenan, Nonthermal Velocities in the Solar Transition Zone and Corona. Solar Phys. 173, 243-258 (1997)

J.G. Doyle, J. Giannikakis, L.D. Xia, M.S. Madjarska, Line broadening of EUV lines across the Solar limb: A spicule contribution? Astron. Astrophys. 431, 17-20 (2005). doi:10.1051/0004-6361:200400137

R. Erdélyi, V. Fedun, Are There Alfvén Waves in the Solar Atmosphere? Science 318, 1572 (2007). doi:10.1126/science.1153006

R. Erdelyi, J.G. Doyle, M.E. Perez, K. Wilhelm, Center-to-limb line width measurements of solar chromospheric, transition region and coronal lines. Astron. Astrophys. 337, 287-293 (1998)

R. Erdélyi, C. Malins, G. Tóth, B. de Pontieu, Leakage of photospheric acoustic waves into non-magnetic solar atmosphere. Astron. Astrophys. 467, 1299-1311 (2007). doi:10.1051/0004-6361:20066857

V. Fedun, R. Erdélyi, S. Shelyag, Oscillatory Response of the 3D Solar Atmosphere to the Leakage of Photospheric Motion. Solar Phys. 258, 219-241 (2009). doi:10.1007/s11207-009-9407-9

A.H. Gabriel, F. Bely-Dubau, P. Lemaire, The Contribution of Polar Plumes to the Fast Solar Wind. Astrophys. J. 589, 623-634 (2003). doi:10.1086/374416

A.H. Gabriel, L. Abbo, F. Bely-Dubau, A. Llebaria, E. Antonucci, Solar Wind Outflow in Polar Plumes from 1.05 to 2.4 R. Astrophys. J. Lett. 635, 185-188 (2005). doi:10.1086/499521

S. Giordano, E. Antonucci, G. Noci, M. Romoli, J.L. Kohl, Identification of the Coronal Sources of the Fast Solar Wind. Astrophys. J. Lett. 531, 79-82 (2000). doi:10.1086/312525

B.E. Goldstein, E.J. Smith, A. Balogh, T.S. Horbury, M.L. Goldstein, D.A. Roberts, Properties of magnetohydrodynamic turbulence in the solar wind as observed by Ulysses at high heliographic latitudes. Geophys. Res. Lett. 22, 3393-3396 (1995). doi:10.1029/95GL03183

M.L. Goldstein, D.A. Roberts, W.H. Matthaeus, Magnetohydrodynamic Turbulence In The Solar Wind. Ann. Rev. Astron. Astrophys. 33, 283-326 (1995). doi:10.1146/annurev.aa.33.090195.001435

G.R. Gupta, E. O'Shea, D. Banerjee, M. Popescu, J.G. Doyle, On the statistical detection of propagating waves in polar coronal holes. Astron. Astrophys. 493, 251-257 (2009). doi:10.1051/00046361:200810602

G.R. Gupta, D. Banerjee, L. Teriaca, S. Imada, S. Solanki, Accelerating Waves in Polar Coronal Holes as Seen by EIS and SUMER. Astrophys. J. 718, 11-22 (2010). doi:10.1088/0004-637X/718/1/11

R.A. Harrison, E.C. Sawyer, M.K. Carter, A.M. Cruise, R.M. Cutler, A. Fludra, R.W. Hayes, B.J. Kent, J. Lang, D.J. Parker, J. Payne, C.D. Pike, S.C. Peskett, A.G. Richards, J.L. Gulhane, K. Norman, A.A. Breeveld, E.R. Breeveld, K.F. Al Janabi, A.J. McCalden, J.H. Parkinson, D.G. Self, P.D. Thomas, A.I. Poland, R.J. Thomas, W.T. Thompson, O. Kjeldseth-Moe, P. Brekke, J. Karud, P. Maltby, B. Aschenbach, H. Bräuninger, M. Kühne, J. Hollandt, O.H.W. Siegmund, M.C.E. Huber, A.H. Gabriel, H.E. Mason, B.J.I. Bromage, The Coronal Diagnostic Spectrometer for the Solar and Heliospheric Observatory. Solar Phys. 162, 233-290 (1995). doi:10.1007/BF00733431

D.M. Hassler, T.G. Moran, Broadening of the FeX (6374 $\backslash \backslash \mathrm{AA}\})$ profiles above the limb in a coronal hole. Space Science Reviews 70, 373-377 (1994). doi:10.1007/BF00777895

D.M. Hassler, G.J. Rottman, E.C. Shoub, T.E. Holzer, Line broadening of MG X 609 and 625 A coronal 
emission lines observed above the solar limb. Astrophys. J. Lett. 348, 77-80 (1990). doi:10.1086/185635

D.B. Jess, M. Mathioudakis, R. Erdélyi, P.J. Crockett, F.P. Keenan, D.J. Christian, Alfvén Waves in the Lower Solar Atmosphere. Science 323, 1582 (2009). doi:10.1126/science.1168680

J.L. Kohl, R. Esser, L.D. Gardner, S. Habbal, P.S. Daigneau, E.F. Dennis, G.U. Nystrom, A. Panasyuk, J.C. Raymond, P.L. Smith, L. Strachan, A.A. van Ballegooijen, G. Noci, S. Fineschi, M. Romoli, A. Ciaravella, A. Modigliani, M.C.E. Huber, E. Antonucci, C. Benna, S. Giordano, G. Tondello, P. Nicolosi, G. Naletto, C. Pernechele, D. Spadaro, G. Poletto, S. Livi, O. von der Lühe, J. Geiss, J.G. Timothy, G. Gloeckler, A. Allegra, G. Basile, R. Brusa, B. Wood, O.H.W. Siegmund, W. Fowler, R. Fisher, M. Jhabvala, The Ultraviolet Coronagraph Spectrometer for the Solar and Heliospheric Observatory. Solar Phys. 162, 313-356 (1995). doi:10.1007/BF00733433

T. Kosugi, K. Matsuzaki, T. Sakao, T. Shimizu, Y. Sone, S. Tachikawa, T. Hashimoto, K. Minesugi, A. Ohnishi, T. Yamada, S. Tsuneta, H. Hara, K. Ichimoto, Y. Suematsu, M. Shimojo, T. Watanabe, S. Shimada, J.M. Davis, L.D. Hill, J.K. Owens, A.M. Title, J.L. Culhane, L.K. Harra, G.A. Doschek, L. Golub, The Hinode (Solar-B) Mission: An Overview. Solar Phys. 243, 3-17 (2007). doi:10.1007/s11207-0079014-6

Y. Lau, E. Siregar, Nonlinear Alfven Wave Propagation in the Solar Wind. Astrophys. J. 465, 451 (1996). doi: $10.1086 / 177432$

Y. Lou, Damping of Low-Frequency Alfvén Waves in Fast Polar Coronal Winds from the Rotating Sun. Astrophys. J. Lett. 571, 187-190 (2002). doi:10.1086/341330

C. Malins, R. Erdélyi, Direct Propagation of Photospheric Acoustic p Modes into Nonmagnetic Solar Atmosphere. Solar Phys. 246, 41-52 (2007). doi:10.1007/s11207-007-9073-8

M.S. Marsh, R.W. Walsh, B.J.I. Bromage, A wavelet analysis of quasi-periodic variability across a solar coronal hole region. Astron. Astrophys. 393, 649-659 (2002). doi:10.1051/0004-6361:20021057

D.J. McComas, B.L. Barraclough, H.O. Funsten, J.T. Gosling, E. Santiago-Muñoz, R.M. Skoug, B.E. Goldstein, M. Neugebauer, P. Riley, A. Balogh, Solar wind observations over Ulysses' first full polar orbit. $J$. Geophys. Res. 105, 10419-10434 (2000). doi:10.1029/1999JA000383

S.W. McIntosh, B. De Pontieu, High-Speed Transition Region and Coronal Upflows in the Quiet Sun. Astrophys. J. 707, 524-538 (2009). doi:10.1088/0004-637X/707/1/524

S.W. McIntosh, D.E. Innes, B. de Pontieu, R.J. Leamon, STEREO observations of quasi-periodically driven high velocity outflows in polar plumes. Astron. Astrophys. 510, 2 (2010). doi:10.1051/0004$6361 / 200913699$

T.G. Moran, Interpretation of coronal off-limb spectral line width measurements. Astron. Astrophys. 374, 9-11 (2001). doi:10.1051/0004-6361:20010643

R. Muller, T. Roudier, J. Vigneau, H. Auffret, The proper motion of network bright points and the heating of the solar corona. Astron. Astrophys. 283, 232-240 (1994)

R.H. Munro, G.L. Withbroe, Properties of a Coronal "hole" Derived from Extreme-Ultraviolet Observations. Astrophys. J. 176, 511 (1972). doi:10.1086/151653

V.M. Nakariakov, L. Ofman, T.D. Arber, Nonlinear dissipative spherical Alfvén waves in solar coronal holes. Astron. Astrophys. 353, 741-748 (2000)

G. Newkirk Jr., J. Harvey, Coronal Polar Plumes. Solar Phys. 3, 321-343 (1968). doi:10.1007/BF00155166

P. Nisenson, A.A. van Ballegooijen, A.G. de Wijn, P. Sütterlin, Motions of Isolated G-Band Bright Points in the Solar Photosphere. Astrophys. J. 587, 458-463 (2003). doi:10.1086/368067

G. Noci, J.L. Kohl, E. Antonucci, G. Tondello, M.C.E. Huber, S. Fineschi, L.D. Gardner, G. Naletto, P. Nicolosi, J.C. Raymond, M. Romoli, D. Spadaro, O.H.W. Siegmund, C. Benna, A. Ciaravella, S. Giordano, J. Michels, A. Modigliani, A. Panasyuk, C. Pernechele, G. Poletto, P.L. Smith, L. Strachan, First results from UVCS/SOHO. Advances in Space Research 20, 2219-2230 (1997). doi:10.1016/S02731177(97)00895-8

L. Ofman, Three-fluid model of the heating and acceleration of the fast solar wind. Journal of Geophysical Research (Space Physics) 109(A18), 7102 (2004). doi:10.1029/2003JA010221

L. Ofman, MHD Waves and Heating in Coronal Holes. Space Science Reviews 120, 67-94 (2005). doi:10.1007/s11214-005-5098-1

L. Ofman, J.M. Davila, Alfvén wave heating of coronal holes and the relation to the high-speed solar wind. J. Geophys. Res. 100, 23413-23426 (1995). doi:10.1029/95JA02222

L. Ofman, J.M. Davila, Do First Results from SOHO UVCS Indicate That the Solar Wind Is Accelerated by Solitary Waves? Astrophys. J. Lett. 476, 51 (1997). doi:10.1086/310491

L. Ofman, J.M. Davila, Three-Fluid 2.5-dimensional Magnetohydrodynamic Model of the Effective Temperature in Coronal Holes. Astrophys. J. 553, 935-940 (2001). doi:10.1086/320960

L. Ofman, V.M. Nakariakov, C.E. DeForest, Slow Magnetosonic Waves in Coronal Plumes. Astrophys. J. 514, 441-447 (1999). doi:10.1086/306944

L. Ofman, V.M. Nakariakov, N. Sehgal, Dissipation of Slow Magnetosonic Waves in Coronal Plumes. Astro- 
phys. J. 533, 1071-1083 (2000). doi:10.1086/308691

L. Ofman, M. Romoli, G. Poletto, G. Noci, J.L. Kohl, Ultraviolet Coronagraph Spectrometer Observations of Density Fluctuations in the Solar Wind. Astrophys. J. Lett. 491, 111 (1997). doi:10.1086/311067

L. Ofman, M. Romoli, G. Poletto, G. Noci, J.L. Kohl, UVCS WLC Observations of Compressional Waves in the South Polar Coronal Hole. Astrophys. J. 529, 592-598 (2000). doi:10.1086/308252

E. O'Shea, D. Banerjee, J.G. Doyle, Magnetoacoustic wave propagation in off-limb polar regions. Astron. Astrophys. 452, 1059-1068 (2006). doi:10.1051/0004-6361:20053687

E. O'Shea, D. Banerjee, J.G. Doyle, A statistical study of wave propagation in coronal holes. Astron. Astrophys. 463, 713-725 (2007). doi:10.1051/0004-6361:20065592

S. Patsourakos, J.C. Vial, Outflow velocity of interplume regions at the base of Polar Coronal Holes. Astron. Astrophys. 359, 1-4 (2000)

M.D. Popescu, D. Banerjee, E. O'Shea, J.G. Doyle, L.D. Xia, Very long period activity at the base of solar wind streams. Astron. Astrophys. 442, 1087-1090 (2005). doi:10.1051/0004-6361:20053714

N. Raouafi, J.W. Harvey, S.K. Solanki, Properties of Solar Polar Coronal Plumes Constrained by Ultraviolet Coronagraph Spectrometer Data. Astrophys. J. 658, 643-656 (2007). doi:10.1086/510286

T. Saito, T. Kudoh, K. Shibata, What Determines the Height of Spicules? I. Alfvén-Wave Model and SlowWave Model. Astrophys. J. 554, 1151-1158 (2001). doi:10.1086/323146

S.R. Spangler, The Amplitude of Magnetohydrodynamic Turbulence in the Inner Solar Wind. Astrophys. J. 576, 997-1004 (2002). doi:10.1086/341889

B.A. Stark, Wave resonances and induced flow due to nonlinear Alfvén waves in a stratified atmosphere. J. Geophys. Res. 101, 15615-15628 (1996). doi:10.1029/96JA00610

T.K. Suzuki, Coronal heating and acceleration of the high/low-speed solar wind by fast/slow MHD shock trains. Mon. Not. Roy. Astron. Soc. 349, 1227-1239 (2004). doi:10.1111/j.1365-2966.2004.07570.x

L. Teriaca, . in preparation (2010)

L. Teriaca, G. Poletto, M. Romoli, D.A. Biesecker, The Nascent Solar Wind: Origin and Acceleration. Astrophys. J. 588, 566-577 (2003). doi:10.1086/368409

H. Tian, L. Xia, Network oscillations at the boundary of an equatorial coronal hole. Astron. Astrophys. 488, 331-337 (2008). doi:10.1051/0004-6361:200810124

S. Tsuneta, K. Ichimoto, Y. Katsukawa, B.W. Lites, K. Matsuzaki, S. Nagata, D. Orozco Suárez, T. Shimizu, M. Shimojo, R.A. Shine, Y. Suematsu, T.K. Suzuki, T.D. Tarbell, A.M. Title, The Magnetic Landscape of the Sun's Polar Region. Astrophys. J. 688, 1374-1381 (2008). doi:10.1086/592226

B.T. Tsurutani, C.M. Ho, J.K. Arballo, B.E. Goldstein, A. Balogh, Large amplitude IMF fluctuations in corotating interaction regions: Ulysses at midlatitudes. Geophys. Res. Lett. 22, 3397-3400 (1995). doi:10.1029/95GL03179

C. Tu, E. Marsch, On the nature of compressive fluctuations in the solar wind. J. Geophys. Res. 99, 21481 (1994). doi:10.1029/94JA00843

C. Tu, E. Marsch, MHD structures, waves and turbulence in the solar wind: Observations and theories. Space Science Reviews 73, 1-210 (1995). doi:10.1007/BF00748891

C. Tu, E. Marsch, K. Wilhelm, W. Curdt, Ion Temperatures in a Solar Polar Coronal Hole Observed by SUMER on SOHO. Astrophys. J. 503, 475 (1998). doi:10.1086/305982

C.Y. Tu, C. Zhou, E. Marsch, L.D. Xia, L. Zhao, J.X. Wang, K. Wilhelm, Solar Wind Origin in Coronal Funnels. Science 308, 519-523 (2005). doi:10.1126/science.1109447

E. Verwichte, V.M. Nakariakov, F.C. Cooper, Transverse waves in a post-flare supra-arcade. Astron. Astrophys. 430, 65-68 (2005). doi:10.1051/0004-6361:200400133

K. Wilhelm, Solar coronal-hole plasma densities and temperatures. Astron. Astrophys. 455, 697-708 (2006). doi:10.1051/0004-6361:20054693

K. Wilhelm, B.N. Dwivedi, L. Teriaca, On the widths of the Mg X lines near $60 \mathrm{~nm}$ in the corona. Astron. Astrophys. 415, 1133-1139 (2004). doi:10.1051/0004-6361:20034234

K. Wilhelm, W. Curdt, E. Marsch, U. Schühle, P. Lemaire, A. Gabriel, J.C. Vial, M. Grewing, M.C.E. Huber, S.D. Jordan, A.I. Poland, R.J. Thomas, M. Kühne, J.G. Timothy, D.M. Hassler, O.H.W. Siegmund, SUMER - Solar Ultraviolet Measurements of Emitted Radiation. Solar Phys. 162, 189-231 (1995). doi:10.1007/BF00733430

K. Wilhelm, E. Marsch, B.N. Dwivedi, D.M. Hassler, P. Lemaire, A.H. Gabriel, M.C.E. Huber, The Solar Corona above Polar Coronal Holes as Seen by SUMER on SOHO. Astrophys. J. 500, 1023 (1998). doi: $10.1086 / 305756$

K. Wilhelm, I.E. Dammasch, E. Marsch, D.M. Hassler, On the source regions of the fast solar wind in polar coronal holes. Astron. Astrophys. 353, 749-756 (2000)

K. Wilhelm, A. Fludra, L. Teriaca, R.A. Harrison, B.N. Dwivedi, C.D. Pike, The widths of vacuum-ultraviolet spectral lines in the equatorial solar corona observed with CDS and SUMER. Astron. Astrophys. 435, 733-741 (2005). doi:10.1051/0004-6361:20042460 
G.L. Withbroe, Evidence for temporal variations in polar plumes. Solar Phys. 89, 77-88 (1983). doi:10.1007/BF00211954

J. Woch, W.I. Axford, U. Mall, B. Wilken, S. Livi, J. Geiss, G. Gloeckler, R.J. Forsyth, SWICS/Ulysses observations: The three-dimensional structure of the heliosphere in the declining/minimum phase of the solar cycle. Geophys. Res. Lett. 24, 2885-2888 (1997). doi:10.1029/97GL01605

Y. Yamauchi, S.T. Suess, T. Sakurai, Relation between Pressure Balance Structures and polar plumes from Ulysses high latitude observations. Geophys. Res. Lett. 29(10), 100000-1 (2002). doi:10.1029/2001GL013820

J. Zhang, J. Woch, S.K. Solanki, R. von Steiger, R. Forsyth, Interplanetary and solar surface properties of coronal holes observed during solar maximum. Journal of Geophysical Research (Space Physics) 108, 1144 (2003). doi:10.1029/2002JA009538 